\title{
A guided search genetic algorithm using mined rules for optimal affective product design
}

\author{
Chris K.Y. Fung ${ }^{1}$, C.K. Kwong ${ }^{1}$, Kit Yan Chan ${ }^{2}$, and H. Jiang ${ }^{1}$ \\ ${ }^{1}$ Department of Industrial and Systems Engineering, The Hong Kong Polytechnic University \\ Hong Kong, PRC \\ ${ }^{2}$ Department of Electrical and Computer Engineering, Curtin University, Western Australia, \\ Australia
}

Corresponding author:

C.K. Kwong (c.k.kwong@polyu.edu.hk)

Department of Industrial and Systems Engineering, The Hong Kong Polytechnic University, Hong Kong

\begin{abstract}
Affective design is an important aspect of new product development, especially for consumer products, to achieve a competitive edge in the marketplace. It can help companies develop new products that can better satisfy the emotional needs of customers. However, product designers usually encounter difficulties in determining the optimal settings of the design attributes for affective design. In this paper, a novel guided search genetic algorithm (GA) approach is proposed to determine the optimal design attribute settings for affective design. The optimisation model formulated based on the proposed approach applied constraints and guided search operators, which were formulated based on mined rules, to guide the GA search and to achieve desirable solutions. A case study on the affective design of mobile phones was conducted to illustrate the proposed approach and validate its effectiveness. Validation tests were conducted, and the results show that the guided search GA approach outperforms the GA
\end{abstract}


approach without the guided search strategy in terms of GA convergence and computational time. In addition, the guided search optimization model is capable of improving GA to generate good solutions for affective design.

Keywords: Affective design, guided search genetic algorithms, new product development, customer satisfaction

\section{Introduction}

Manufacturers currently face a highly competitive environment as consumers become more demanding because of the availability of more choices in the market as a result of globalization. However, product technology has become more sophisticated and accessible, and this trend is gradually reducing the marginal value of adding new functions to products. Today, customers consider functionality, ease of use, and reliability as product requirements, such that the design for performance and the design for usability can no longer guarantee a competitive advantage (Liu, 2003). Aside from these tangible product aspects, customers also consider intangible and emotional aspects, such as metaphors, novelty, personality, aesthetics, and style of products (Crilly et al., 2004; Demirbilek and Sener, 2003). Design attributes, such as form and color, evoke the affective responses of customers to products, as well as attract them and influence their choices and preferences, such as loyalty and joy of use (Creusen and Schoormans, 2005; Noble and Kumar, 2008). The satisfaction of the emotional needs of customers is synonymous to the 'feeling quality' of the products (Lai et al., 2005a). The affective design involves activities that identify, measure, analyze, and understand the relationship between affective needs in the customer domain and perceptual design attributes in the design domain. The affective design provides decision support for design optimization, such that appealing products that satisfy the emotional needs of target customers can be developed successfully (Jiao et al., 2006; Khalid and Helander, 2004). Therefore, affective design is important for customeroriented and market-driven product development because it helps increase customer satisfaction 
and adds value to products, such as automobiles, furniture, cosmetic containers, and many other products used in daily life (Catalano, 2002; Nagamachi, 2002).

Kansei Engineering, proposed by Nagamachi (1995), is a product development methodology of acquiring and transforming customer affection into design attribute settings with the use of quantitative methods. 'Kansei' is a Japanese word that means psychological feelings, sensations, and emotions. The framework of KE encompasses four tasks (Barnes and Lillford, 2007, 2009; Nagamachi, 2008; Schütte and Eklund, 2005): definition of the product domain, determination of the dimensions of customer affection, determination of design attributes and attribute options, and evaluation of relations between customer affection and design attributes. However, Kansei engineering is unable to determine the optimal design attribute settings for affective design.

A number of studies have been conducted on affective design, and most of them focused on the relationships between affective responses and design attributes with the use of various techniques, such as logistic regression (Barone, 2007), association rule mining (Jiao, 2006), grey theory (Hsiao, 2002), artificial neural networks (Lin, 2008), and neural fuzzy networks (Park, 2004 and Kwong et al., 2009). However, only a few studies focused on determining the optimal design attribute settings for affective design. The main goal in affective design is to determine the optimal settings of design attributes for the affective aspects of products to achieve maximum customer satisfaction. Therefore, affective design can be transformed into a single-objective optimization problem if the optimization focuses on investigating the design utility for a particular customer affection or product image. Various optimisation techniques can be applied, such as linear programming and nonlinear programming (Mishra, et al., 2013). Aktar et al. (2009) employed linear and nonlinear programming to obtain the optimal design attribute settings for affective design. An alternative approach to design optimization is based 
on heuristic algorithms, such as GAs and simulated annealing (Velasco, et al., 2012; Kaplan and Rabadi, 2013), which are stochastic and effective optimization techniques to search for near-optimal solutions for various problems of engineering design (Saridakis and Dentsoras, 2008; Chakraborty et al., 2003). GAs in particular have been applied in various areas of product design, such as product planning (Jiao et al., 2007; D'Souza, 2003), interactive generative design (Kim and Cho, 2000; Yanagisawa and Fukuda, 2005), and optimization of affective design (Hsiao and Liu, 2004; Hsiao and Tsai, 2005; Jiao et al., 2008; Yang and Shieh, 2010). GAs are suitable to solve optimization problems for affective design because of three reasons (Jiao et al., 2008). First, discrete attributes are commonly used in affective design. Compared with traditional optimization techniques, GAs perform better in solving combinatorial optimization problems that involve discrete attributes (Jiao et al., 2007). Second, the optimization problems of affective design are different from many problems of engineering design in which optimal solutions exist. Third, GAs offer good compatibility with different models, whether these are statistical, rule based, or 'black-box' models (Saridakis and Dentsoras, 2008).

In this paper, a novel guided search GA approach using mined rules is proposed to generate the optimal design attribute settings for affective design. The proposed optimization model applied constraints and guided search operators, which were formulated based on mined rules, to guide the GA search and to achieve desirable solutions. A case study on the affective design of mobile phones was conducted to illustrate the proposed approach and the development of the intelligent system, as well as to validate their effectiveness. Results show that the guided search GA approach outperforms the GA approach without the guided search strategy in terms of better solution quality and shorter computational time. 


\section{Optimal affective design problem}

The relationship between affective response and design attributes in affective design is given by the following:

$$
y_{k}=f_{k}\left(x_{1}, x_{2}, \ldots, x_{N}\right)
$$

where $y_{k}$ is the satisfaction value of affective dimension $k$, and $x_{j}$ is the $j$-th design attribute of the product with $j=1,2, \ldots N$.

Model (1) is commonly developed based on customer survey data. Various techniques such as statistical regression (Kuang and Jiang, 2008), neural networks (Chen et al., 2006), and neural-fuzzy networks (Kwong et al., 2009) have been attempted to develop the model. Product designers intend to determine the optimal design attribute settings of an affective design such that the sum or weighed sum of satisfaction values of various affective dimensions are maximized. The optimization problem can be expressed as follows:

$$
\begin{aligned}
& \max : \sum_{k=1}^{M} w_{k} \cdot y_{k} \\
& \text { s.t. } \\
& y_{k}=f_{k}\left(x_{1}, x_{2}, \ldots, x_{N}\right) \\
& x_{j} \in\left(x_{j}^{a}, x_{j}^{b}\right)
\end{aligned}
$$

Where $w_{k}$ is the importance weight of the affective dimension $\mathrm{k}$ and $\left(x_{j}^{a}, x_{j}^{b}\right)$ is the range of value setting of $x_{j}$.

\section{Guided search genetic algorithms for affective design optimization}

A guided search GA approach is proposed to determine the optimal design attribute settings for affective design. The approximate rules mined from customer survey data are used to guide the 
GA search for the optimal design attribute settings. First, ranges of the design attributes are specified onto the guided search GA, and a population of chromosomes is generated randomly with respect to the specified ranges. Apart from using the traditional genetic operations, such as crossover and mutation, to evolve the chromosomes, a guided search operation is developed based on the constraint rules to improve convergence speech and enhance the ability of the chromosomes to locate the global optimum. A fitness function embedded with the predictive model (1) and constraint rules are developed to evaluate the satisfaction of affective dimensions represented by the chromosomes. Figure 1 shows the architecture of the guided search GAbased design optimization model for affective design. The detailed mechanisms of the guided search GA approach, including chromosome representation, crossover and mutation, fitness function, guided search operation, and ranking and selection of the next generation, are given in the following sections.

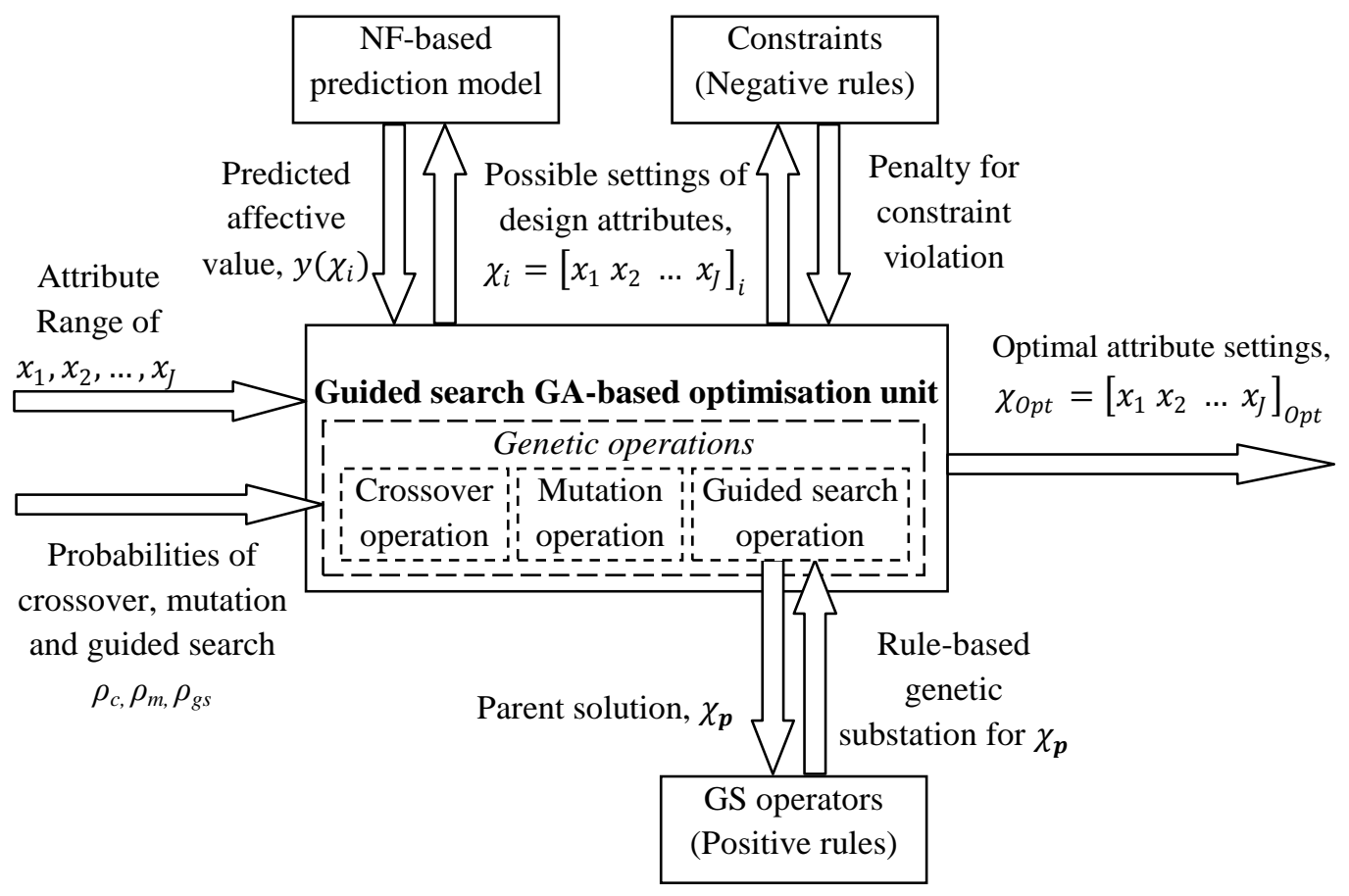


Figure 1 Architecture of the guided search GA-based optimization model.

\subsection{Chromosome representation}

A population of chromosomes is initialized randomly at the start of the evolutionary process, in which each chromosome is used to represent the categorical and quantitative design attributes of the product illustrated in Figure 2. In each chromosome, the genes represent the $(P+Q)$ design attributes of which the product has $P$ categorical design attributes (namely, $A_{i}$ with $i=1,2, \ldots, P$ ) and $Q$ quantitative design attributes (namely, $A_{j}$ with $j=1+P, 2+P, \ldots, P+Q$ ). The 1 st to the $p$-th genes represent $A_{i}$ with $i=1,2, \ldots, P$, and the $(P+1)$-th to the $(P+Q)$-th genes represent $A_{j}$ with $j=1,2, \ldots, Q$.

\begin{tabular}{|c|c|c|c|c|c|c|}
\hline \multirow{2}{*}{$\begin{array}{l}\text { Attribute } \\
\text { Type } \\
\text { Attributes }\end{array}$} & \multicolumn{3}{|c|}{ Categorical Attributes } & \multicolumn{3}{|c|}{ Quantitative Attributes } \\
\hline & $\begin{array}{c}A_{p} \\
\left(x_{1}\right)\end{array}$ & $\begin{array}{l}\cdots \\
\cdots\end{array}$ & $\begin{array}{c}A_{P} \\
\left(x_{P}\right)\end{array}$ & $\begin{array}{c}A_{q} \\
\left(x_{P+1}\right)\end{array}$ & $\begin{array}{l}\cdots \\
\cdots\end{array}$ & $\begin{array}{c}A_{Q} \\
\left(x_{P+Q}\right)\end{array}$ \\
\hline Code type & Integer & Integer & Integer & Real number & Real number & Real number \\
\hline Range & {$\left[1, L_{p}\right]$} & $\cdots$ & {$\left[1, L_{P}\right]$} & {$\left[v_{q}^{\min }, v_{q}^{\max }\right]$} & $\cdots$ & {$\left[v_{Q}^{\min }, v_{Q}^{\max }\right]$} \\
\hline
\end{tabular}

Figure 2 Combined chromosome structure for the design optimization process.

For the categorical design attribute, an integer-code genome is used to represent the selected categorical option within an attribute domain. The range of the integer-code gene is from 1 to $L_{i}$, where the categorical design attribute $A_{i}$ contains $L_{i}$ items with $i=1, \ldots, p$. For quantitative attributes, a real-coded gene is applied to represent the continuous value, which is limited by the range of $\left[v_{j}^{\min }, v_{j}^{\max }\right]$, where $v_{j}^{\min }$ and $v_{j}^{\max }$ are the minimum and maximum values of $A_{j}$ with $j=(1+P), \ldots,(P+Q)$. 


\subsection{Crossover and mutation}

Two different mechanisms for the crossover and mutation are used to reproduce the integercoded and real-coded genes that represent the categorical and quantitative attributes, respectively. Figure 3 shows that for the integer-coded genes, a two-point crossover is used to swap the genes of two parent chromosomes between two random points and reproduce child chromosomes. Figure 4 shows that mutation is used to introduce random values on some randomly selected genes. For real-coded genes, simulated binary crossover and polynomial mutation are used (Agrawal et al., 1995; Deb et al., 2002; Sivakumar, 2012). In polynomial mutation, a gene, $x_{i}$, is randomly selected, and its mutated value, $x_{i}{ }^{\prime}$, is given by the following formulation:

$$
x_{i}{ }^{\prime}=x_{i}+\left(v_{i}^{\max }-v_{i}^{\min }\right) \cdot \delta
$$

where $v_{i}^{\max }$ and $v_{i}^{\min }$ are the upper and lower bounds of $x_{i}$, respectively, and $\delta$ is the random variable given by the polynomial distribution formulated as

$$
\begin{array}{ll}
\delta=(2 \xi)^{\frac{1}{n_{m}+1}}-1, & \text { if } \xi<0.5 ; \\
\delta=1-[2(1-\xi)]^{\frac{1}{n_{m}+1}}, & \text { if } \xi>0.5 ;
\end{array}
$$

$\eta_{m}$ is a mutation distribution index, and $\xi$ is randomly generated between 0 and 1.

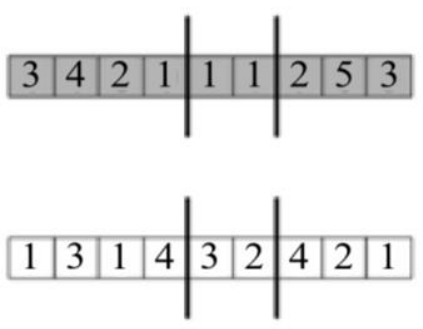

Figure 3 Two-point crossover for both integer-coded and real-coded genes. 


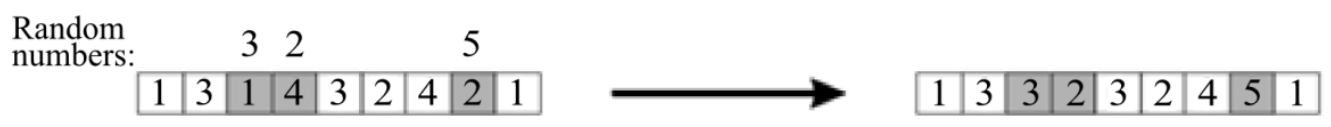

Figure 4 Mutation for integer-coded genes.

\subsection{Fitness function}

The fitness function adopted in this research is defined as follows.

$$
\operatorname{Fit}\left(\chi_{i}\right)=\sum_{k=1}^{M}\left[\psi_{\max }-f_{k}\left(\chi_{i}\right)\right] w_{k}
$$

where $f_{k}\left(\chi_{i}\right)$ is the predicted satisfaction value of the affective dimension $k$ of the neuralfuzzy model; $\psi_{\max }$ is the maximum affective level based on the rating scale; $w_{k}$ is the importance weight of the affective dimension $k$ and and $\sum w_{k}$ is equal to 1 ; and $\operatorname{Fit}\left(\chi_{i}\right)$ is the fitness value based on the chromosome $\chi_{i}$.

With the fitness function, the genetic algorithm would search for the design attribute setting which yields the predicted satisfaction values of various affective dimensions closest to the maximum affective satisfaction value. Therefore, the fitness with a small value is better than that with a large value. However, the time of convergence may be too slow, and the global optimum may not be likely reached if the search of the optimal solution is solely based on the fitness function. Two performance measures, namely, violation $N_{v i o}\left(\chi_{i}\right)$ and penalty $\operatorname{Pen}_{\text {sum }}\left(\chi_{i}\right)$, are thus introduced to reduce the convergence time and increase the chance of searching the global optimum. 
$N_{v i o}\left(\chi_{i}\right)$ is defined as the number of negative rules that the design attribute setting represents by matching $\chi_{i}$ with the "IF" part of the negative rule. However, the affective response does not match the "THEN" part of the negative rule. The penalty value, $\operatorname{Pen}_{\text {sum }}\left(\chi_{i}\right)$, is given by

$$
\begin{gathered}
\operatorname{Pen}_{\text {sum }}\left(\chi_{i}\right)=\sum_{n=1}^{N_{\text {vio }}\left(\chi_{i}\right)} \operatorname{Pen}_{n}\left(\chi_{i}\right) \\
\operatorname{Pen}_{\mathrm{n}}\left(\chi_{i}\right)=\left\{\begin{aligned}
w_{\left(R_{\mathrm{n}}^{-}\right)} \times C F_{\left(R_{n}^{-}\right)} \times\left|\left(\frac{\psi_{L\left(R_{n}^{-}\right)}}{\psi_{\max }-\psi_{\min }}\right)-y\left(\chi_{i}\right)\right|, & \text { if } \chi_{i} \text { violate } \mathrm{trth} \text { constraint } \\
0, & \text { if not }
\end{aligned}\right.
\end{gathered}
$$

where $w_{\left(R_{\mathrm{n}}^{-}\right)}$and $C F_{\left(R_{\mathrm{n}}^{-}\right)}$are the weight and confidence factor of rule $R_{\mathrm{n}}^{-}$, respectively, $\Psi_{L\left(R_{\mathrm{n}}^{-}\right)}$is the affective response described in the "THEN" part of rule $R_{\mathrm{n}}^{-}$, and $\psi_{\min }$ and $\psi_{\max }$ are the minimum and maximum of the affective levels based on the rating scale, respectively. Based on the penalty function, the fitness of the chromosome is downgraded significantly when the consequences of the "IF" part and the "THEN" part do not match. As a result, the design attribute settings causing undesired affective responses are not considered in the optimization.

\subsection{Guided search operation}

A fraction of population in each generation is selected to perform the guided search operation, which intends to search for the process of locating the global optima. The number of chromosomes selected to perform the guided search operation is less than that selected to perform crossover and mutation; otherwise, population diversity is difficult to maintain. The 
positive rules in the rule set are adopted for the guided search operators. The preferred design attribute values stated in the "IF" part of the positive rule are substituted into some of the genes. This process helps obtain a good and preferred solution within a short time. When a chromosome $\chi_{i}$ is selected to be performed with the guided search operation, $N_{g s}$ children $\chi_{g}^{c}$, with $g=1,2, \ldots, N_{g s}$ is generated by shaking $\chi_{i}$ for $N_{g s}$ time. The values of some genes of $\chi_{i}$ are replaced by the design attributes represented in the "IF" part of the rule. For the parts of categorical attributes, integer-code genes are shaken, as shown in Figure 4:

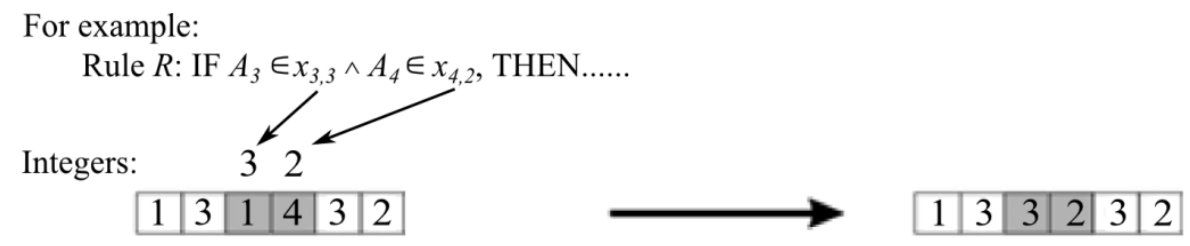

Figure 4 Guided search operator for the integer-coded genes.

For the parts of the quantitative attributes, the new value of the $i$-th gene $x_{g, i}^{c}$ in $\chi_{g}^{c}$ is shaken as

$$
x_{g, i}^{c}=\gamma \times\left(v_{i}^{\max }-v_{i}^{\min }\right)+v_{i}^{\min },
$$

where $\gamma$ donates a random number generated between $[0,1]$. After all $\chi_{g}^{c}$ with $g=1,2, \ldots, N_{g s}$ are reproduced, their fitness Fit $\left(\chi_{g}^{c}\right)$, violation $N_{v i o}\left(\chi_{g}^{c}\right)$, and penalty $\operatorname{Pen}_{\text {sum }}\left(\chi_{g}^{c}\right)$ are determined. The best child $\chi^{\text {best }}$ among the children can be found by ranking them in ascending order according to their violation, penalty, and fitness value. $\chi_{i}$ is replaced with $\chi^{\text {best }}$, if $F i t\left(\chi^{\text {best }}\right)<F i t\left(\chi_{i}\right)$. Otherwise, the substitution is withdrawn if Fit $\left(\chi_{i}\right)<$ Fit $\left(\chi^{\text {best }}\right)$. The pseudo-code of the guided search operation is shown below. 
Randomly select one $\chi_{i}$ in the population as parent solution, $\chi_{\boldsymbol{p}}$

FOR $g=1$ to $N_{g s} / /$ where $N_{g s}$ is the number of GS operators formulated

Reproduce $\chi_{p}$ as child solution $\chi_{c, g}$ using $g$ th GS operator

Evaluate the fittest of objective function, Fit $\left(\chi_{c, g}\right)$, for solution $\chi_{c, g}$

IF solution $\chi_{\boldsymbol{c}, \boldsymbol{g}}$ violates any constraints,

Find $N_{v i o}\left(\chi_{c, g}\right)$

Calculate $P$ en $n_{\text {sum }}\left(\chi_{i}\right)$

\section{END IF}

\section{END FOR}

Rank all children based on smaller $\left\{N_{v i o}\left(\chi_{\boldsymbol{c}, \boldsymbol{g}}\right), \operatorname{Pen}_{\text {sum }}\left(\chi_{i}\right), \operatorname{Fit}\left(\chi_{\boldsymbol{c}, \boldsymbol{g}}\right)\right\}$

Choose child ranked first as the best child $\chi_{c}^{\text {best }}$

// compare the fittest of objective function between the best child and parent

$\operatorname{IF~Fit~}\left(\chi_{c}^{\text {best }}\right)<\operatorname{Fit}\left(\chi_{p}\right)$,

$/ /$ return the best child if $\chi_{c}^{\text {best }}$ is better than $\chi_{\boldsymbol{p}}$

RETURN $\chi_{c}^{\text {best }}$

ELSE

\section{RETURN $\chi_{p}$}

END IF

\subsection{Ranking and selection of the next generation}

The ranking processes involve the three measures, namely, $F i t\left(\chi_{i}\right), N_{\text {vio }}\left(\chi_{i}\right)$, and $P e n_{\text {sum }}\left(\chi_{i}\right)$,

whereas the population is classified into two groups, the desirable and undesirable group. Candidate solutions are in the desirable group if they do not satisfy constraint violations, i.e., $N_{\text {vio }}\left(\chi_{i}\right)=0$. They are ranked according to their fitness value $F i t\left(\chi_{i}\right)$ when they are in the 
desirable group. When they are in the undesirable group, they are ranked based on $N_{\text {vio }}\left(\chi_{i}\right)$ and $\operatorname{Pen}_{\text {sum }}\left(\chi_{i}\right)$. For candidate solutions that violate any constraint $N_{\text {vio }}\left(\chi_{i}\right) \geq 1$, their priorities are based on parameters $N_{\text {wio }}\left(\chi_{i}\right)$ and $P e n_{\text {sum }}\left(\chi_{i}\right)$. For example, let $\chi_{1}$ and $\chi_{2}$ be two different solution vectors. $\chi_{1}$ dominates $\chi_{2}$ if $N_{\text {vio }}\left(\chi_{1}\right)<N_{\text {vio }}\left(\chi_{2}\right)$. If $N_{\text {vio }}\left(\chi_{1}\right)=N_{\text {vio }}\left(\chi_{2}\right)$, the sum of the penalties of $\chi_{1}$ and $\chi_{2}$ is compared, and $\chi_{1}$ dominates $\chi_{2}$ if $\operatorname{Pen}_{\text {sum }}\left(\chi_{1}\right)<\operatorname{Pen}_{\text {sum }}\left(\chi_{2}\right)$.

For each generation, the pool of chromosomes is formed by recombination of the current population (parents) and the reproduced children. Child chromosomes are generated until the pool size is equal to $N_{\text {pop }} \times 2$. The selection process is performed after the preparation of the pool of chromosomes. Binary tournament selection method is adopted for the guided search GA because this method is an effective selection operator of GA (Deb et al, 2002). Two solutions are randomly selected from the pool of chromosomes, and the better one is selected as the offspring of the next generation. The selection is repeated until the population size of the new generation reaches $N_{\text {pop }}$.

Figure 6 shows the mechanisms of the guided search GA. 


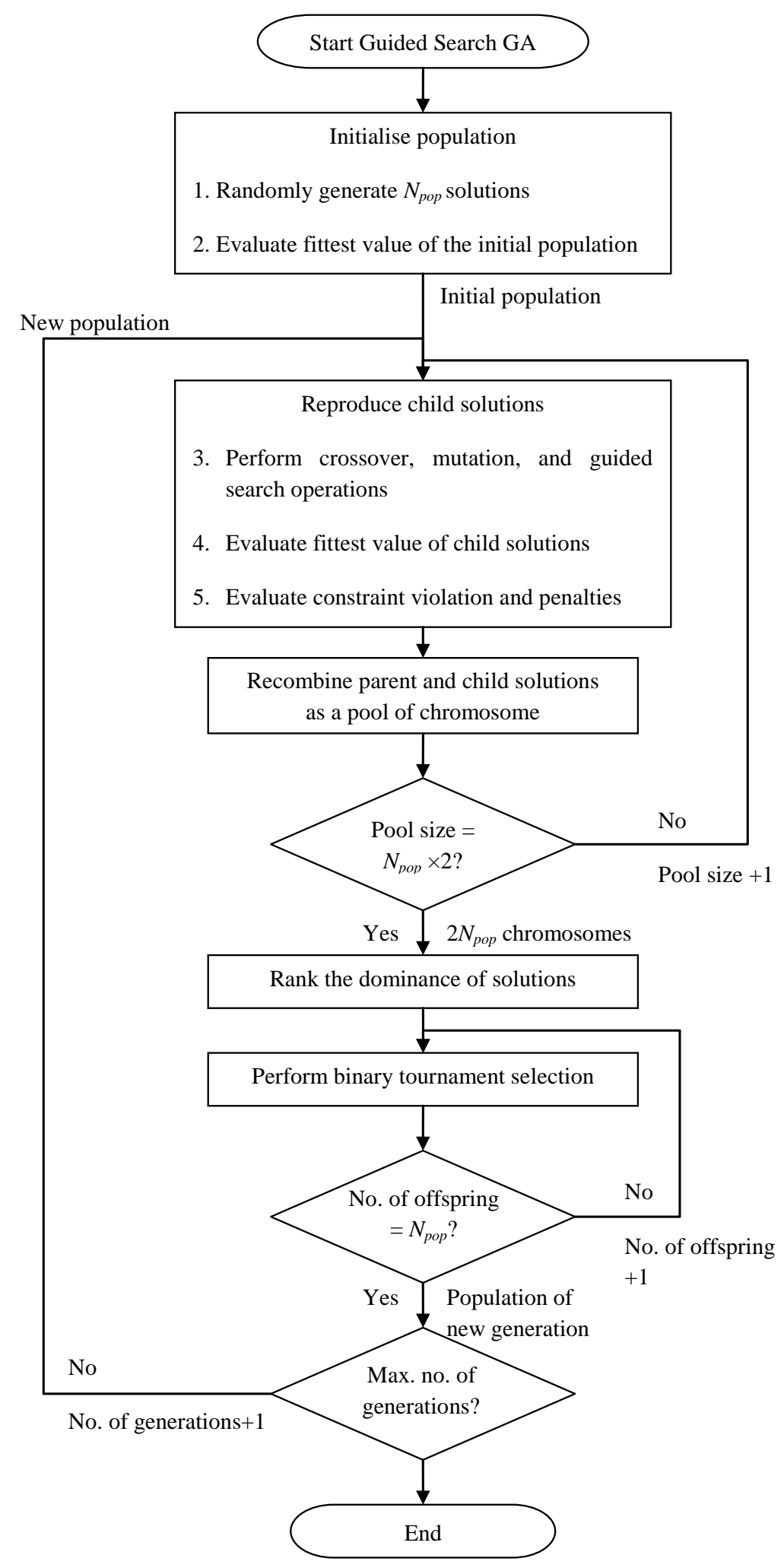

Figure 6 Flowchart of the guided search GA. 


\section{A Case study on the optimal affective design for mobile phones}

A case study on the product form design for mobile phones is used to investigate the effectiveness of the proposed guided search GA for affective design. The case study mainly involves a survey and the implementation of the proposed approach for the affective design for mobile phones. The guided search GA was implemented with MATLAB software programming language. The survey was conducted using questionnaires. It involved customer affections on 32 mobile phone samples based on four product attributes: simplicity, uniqueness, high technology, and handiness. Figure 7 shows the front and side views of the 32 mobile phone samples. Figure 8 shows that a total of 34 respondents filled out the questionnaires and indicated their feelings toward the product images of each sample on a five-point scale.

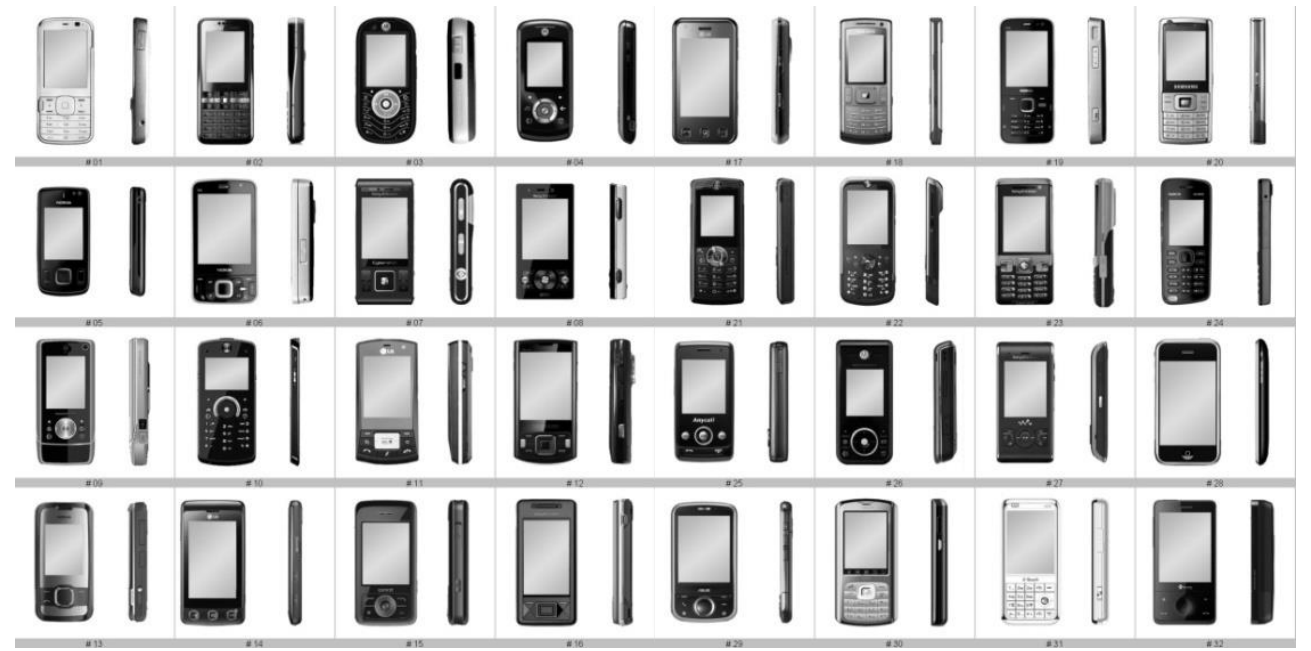

Figure 7 The 32 mobile phone samples used in the case study. 

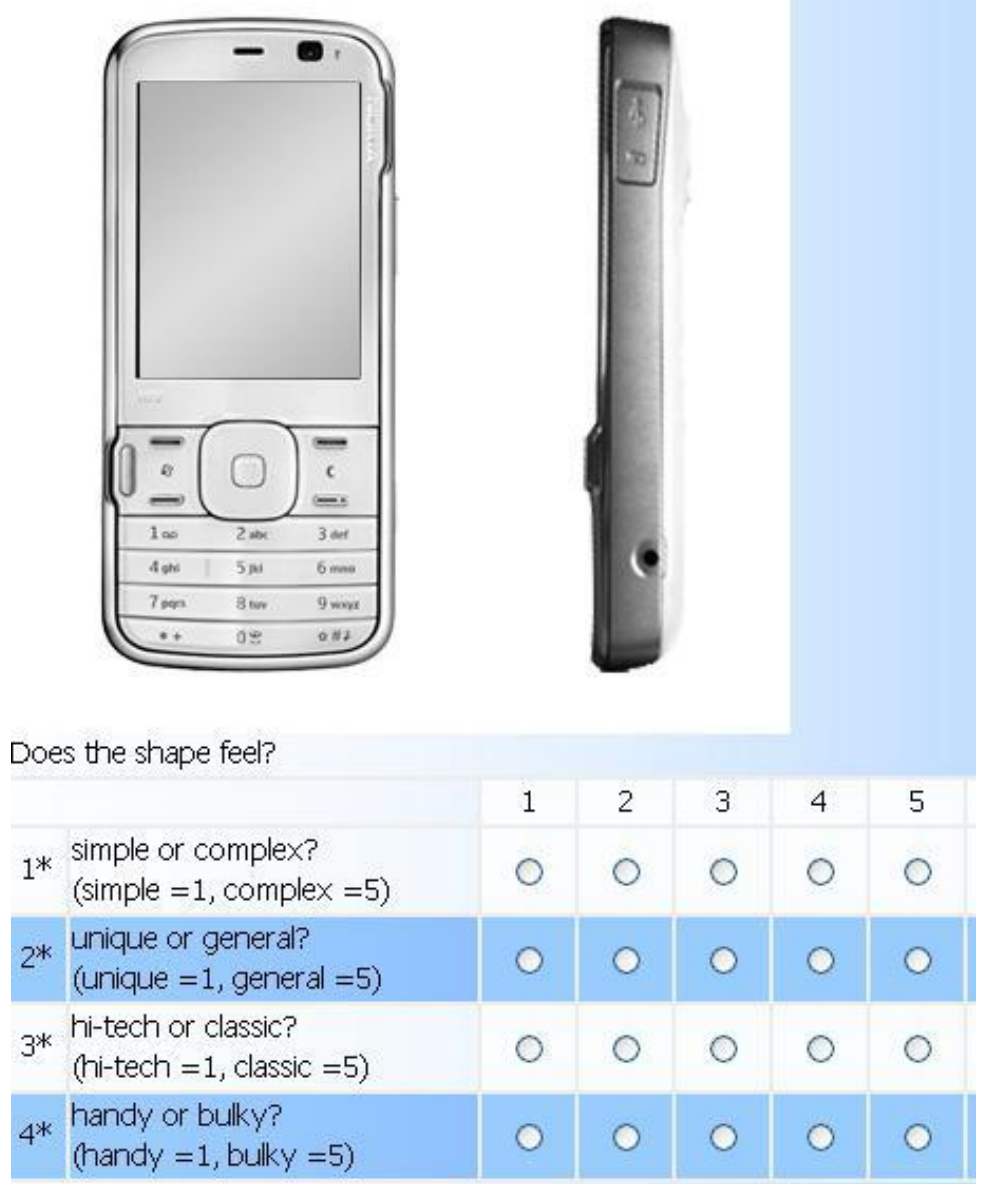

Figure 8 Format of the questionnaire for each mobile phone.

The morphological approach was adopted to define the design space of the product form for mobile phones. Depicting the design composition and possible design solutions with simple and graphical notations was feasible. Eight design parameters $\left(A_{1}\right.$ to $\left.A_{8}\right)$ were defined to describe the product forms of the mobile phones, including top shape, bottom shape, function button shape, layout, length, width ratio, thickness, and border width. The first four design parameters are categorical, whereas the remaining four attributes are quantitative. The categorical attributes contain three to five options. Table 1 shows that the design attributes and their options are listed in a design table for the product form of the mobile phones. Based 
on the design table, the design profile for each sample was identified, and the values of the design attributes can be measured.

Table 1 Design table for the product form of the mobile phones

\begin{tabular}{|c|c|c|c|c|c|c|}
\hline \multicolumn{7}{|c|}{ Categorical Attributes } \\
\hline & Elements & Type 1 & Type 2 & Type 3 & Type 4 & Type 5 \\
\hline 1. & $\begin{array}{l}\text { Top Shape } \\
\qquad\left(A_{1}\right)\end{array}$ & $\begin{array}{l}\text { Line } \\
\left(x_{11}\right)\end{array}$ & $\begin{array}{l}\operatorname{Arc} \\
\left(x_{12}\right)\end{array}$ & $\begin{array}{c} \\
\text { Curve } \\
\left(x_{13}\right)\end{array}$ & & \\
\hline 2. & $\begin{array}{l}\text { Bottom Shape } \\
\quad\left(A_{2}\right)\end{array}$ & $\begin{array}{l}\text { Line } \\
\left(x_{21}\right) \\
\end{array}$ & $\begin{array}{l}\text { Arc } \\
\left(x_{22}\right) \\
\end{array}$ & $\begin{array}{c}\text { Curve } \\
\left(x_{23}\right)\end{array}$ & & \\
\hline 3. & $\begin{array}{c}\text { Function Button } \\
\text { Shape } \\
\left(A_{3}\right)\end{array}$ & $\begin{array}{c}(0) \\
\substack{\text { Large round } \\
\left(x_{31}\right)}\end{array}$ & $\begin{array}{c}(0) \\
\text { Small round } \\
\left(x_{32}\right)\end{array}$ & $\begin{array}{c}\square \\
\text { Small squares } \\
\left(x_{33}\right)\end{array}$ & $\begin{array}{c}\text { Large square } \\
\left(x_{34}\right)\end{array}$ & $\begin{array}{c}\square \\
\text { Wide Block } \\
\left(x_{35}\right)\end{array}$ \\
\hline 4. & $\begin{array}{l}\text { Layout } \\
\left(A_{4}\right)\end{array}$ & 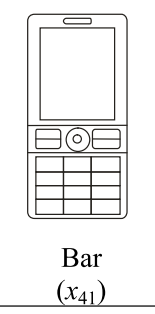 & 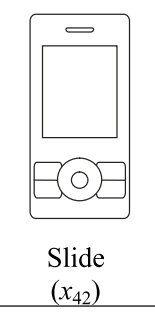 & \begin{tabular}{c|} 
\\
\\
\\
$\square \odot \square$ \\
Large screen \\
$\left(x_{43}\right)$
\end{tabular} & & \\
\hline \multicolumn{7}{|c|}{ Quantitative Attributes } \\
\hline 5. & $\begin{array}{l}\text { Body Length } \\
\qquad\left(A_{5}\right)\end{array}$ & & & & & \\
\hline 6. & $\begin{array}{l}\text { Body Width } \\
\qquad\left(A_{6}\right)\end{array}$ & & & & & \\
\hline 7. & $\begin{array}{c}\text { Body Thickness } \\
\left(A_{7}\right)\end{array}$ & & & & & \\
\hline 8. & $\begin{array}{l}\text { Border Width } \\
\quad\left(A_{8}\right)\end{array}$ & & & & & \\
\hline
\end{tabular}




\subsection{Implementation of the guided search GA}

The optimal settings of the design attributes for the affective design of mobile phones are determined through the development of a guided search GA optimization model, as shown in Figure 1. The neural-fuzzy model for modeling affective relationships, which was developed by the authors in their previous research (Kwong, 2009), was adopted as the predictive model. Based on multi-objective GA (MOGA) approach, the approximate rules were mined from the customer survey data. Table 2 shows the approximate rules for the affective dimension 'Handiness' determined based on the MOGA-based rule mining approach. The details of the rule mining using MOGA approach are detailed in the authors' previous study (Fung, 2012). In this case study, the proposed guided search GA approach was implemented with MATLAB software programming language to maximize the "handiness" of mobile phone design. The fitness function of the GA (8) is the "the-smaller-the-better" function, so the threshold of the target value was set to $1 \times 10^{-5}$ as the stopping criteria of the GA optimization.

Four different GA optimization strategies were used to investigate the performance of the proposed guided search GA. These include the non-guided search strategy ("No GS"), three guided search strategies using all rules (“GS All R"), only positive rules (“GS +ve R”), and only negative rules (“GS -ve R"). The test on No GS executed the non-constrained GA and no guided search operators. By contrast, GS All R applied the constraints and facilitated the search operators to thoroughly guide the GA search. GS + ve R adopted only the guided search operators defined by positive rules, as shown in Table 3. GS -ve R performed the constrained GA with the penalty approach, and the constraints were defined based on the negative rules shown in Table 3. GS +ve R and GS -ve R were used to investigate the performance of the partially guided search approach when the rule set is incomplete or contains either positive or negative rules only. 
Table 2 Approximate rules obtained based on the MOGA-based rule-mining method

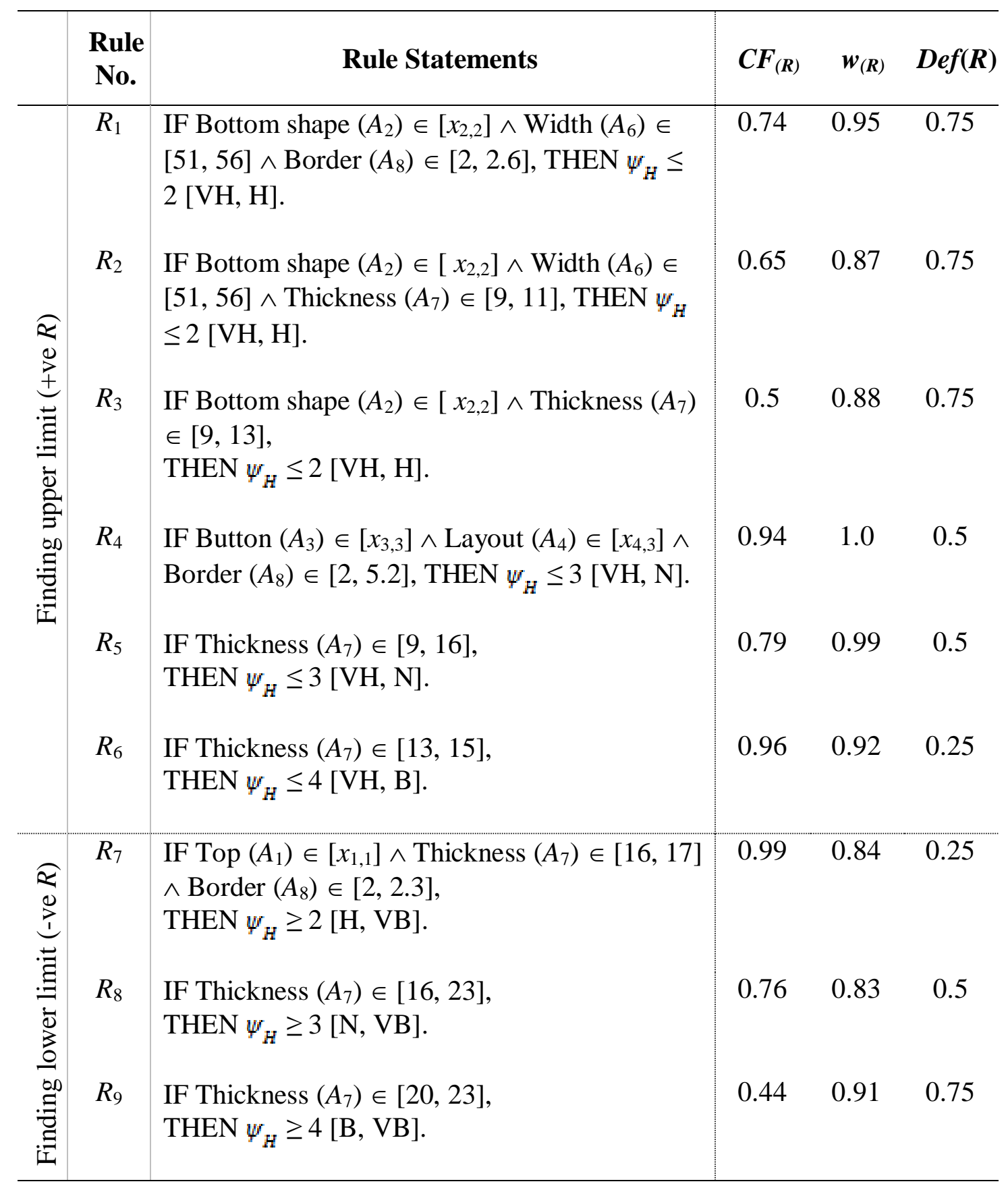

where $\psi_{H}$ is the rule approximation for the handiness of mobile phones, $\mathrm{VH}$ is very handy $\left(\psi_{H}=1\right), \mathrm{H}$ is handy $\left(\Psi_{H}=2\right), \mathrm{N}$ is normal $\left(\psi_{H}=3\right), \mathrm{B}$ is bulky $\left(\psi_{H}=4\right)$, and VB is very bulky $\left(\psi_{H}=5\right)$.

Inequity is avoided with the same initial population for the preference test of all four optimization strategies instead of the population from random initialization. This measure ensures that all GA convergences begin from the same starting point. For each generation, the 
best fitness value of the population was evaluated with a population size of 50. For the tests of No GS and GS -ve R, $\rho_{c}$ and $\rho_{m}$ are set to $75 \%$ and $25 \%$, respectively, and the guided search operators are disabled $\left(\rho_{g s}=0 \%\right)$. For GS All R and GS +ve R, the guided search operators were enabled, and $\rho_{c}, \rho_{m}$, and $\rho_{g s}$ are set to $50 \%, 25 \%$, and $25 \%$, respectively.

\subsection{Results of design optimization}

Figure 9 shows the GA convergence results of the four GA optimization strategies in which the proposed approach converges faster than the non-guided search approaches (No GS). All guided search GAs start at the same point where the best and mean fitness values of the initial population are 0.25 and 0.4 , respectively. After 100 generations, the best fitness value gradually decreased from 0.25 to below 0.1 with the No GS approach. By contrast, convergence was dramatically improved by the GS All R approach. The mean fitness value of the population was minimized to nearly zero (less than 0.01 ), and the near-optimal solution was almost found by the GS All R approach after 60 generations. The GS +ve R and GS -ve $\mathrm{R}$ approaches also provided notable improvements to GA convergence. However, employing either GS operators or constraints cannot guide the search as effectively as the GS All R search. The GS -ve R approach only slightly impelled the convergence compared with the No GS approach. GS +ve R remarkably accelerated convergence in the first 30 generations. However, convergence became sluggish, and the fitness value was held above 0.5. The GS All R approach yielded the best GA convergence among the four optimization models. 


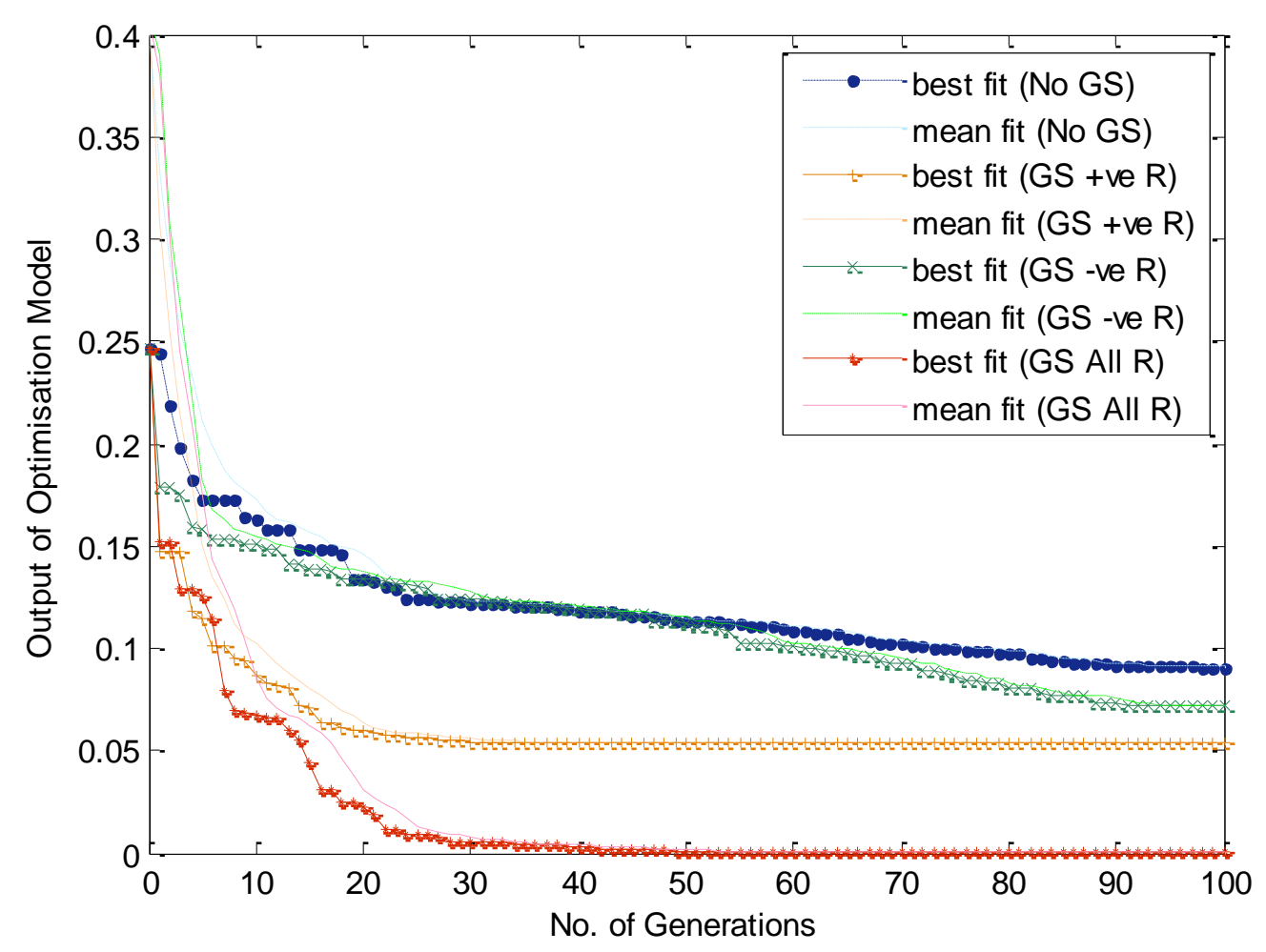

Figure 9 Comparison among the convergences of the guided search GAs

The computational times between the guided search and non-guided search approaches were compared. Table 3 shows the computational times of the four optimization strategies. Six guided search operators were defined, and their operating rate $\rho_{g s}$ was set to 25\%. Table 4 shows three constraints that were set. The computational time of the GS -ve R model only required $0.4 \%$ more than that of the No GS model in terms of average computational time per generation. The GS All R and GS +ve R models required about $40 \%$ more computational time per generations than the No GS model. Additional computational time was required for the objective function evaluation and selection of temporary solutions produced by the guided search operators. However, the superior searching ability of the GS All R model overcame this problem. The GS All R model reached the target fitness with the minimum number of generations and with the shortest total computation time. 
Table 3 Comparison of the computational time of the guided search genetic algorithms

\begin{tabular}{ccccc}
\hline $\begin{array}{c}\text { GA } \\
\begin{array}{c}\text { Optimisation } \\
\text { Strategy }\end{array}\end{array}$ & $\begin{array}{c}\text { No. of } \\
\text { Generations } \\
\text { Elapsed }\end{array}$ & $\begin{array}{c}\text { Total Computational Average Time per } \\
\text { Time Used / second } \\
\text { Generations / } \\
\text { second }\end{array}$ & $\begin{array}{c}\text { Best Fitness } \\
\text { (Minimising) }\end{array}$ \\
\hline 'No GS' & 301 & 20.02 & 0.0665 & 0.1844 \\
'GS +ve R' & 213 & 20.05 & 0.0941 & 0.0540 \\
'GS -ve R' & 300 & 20.03 & 0.0668 & 0.0779 \\
'GS All R' & 130 & 12.20 & 0.0938 & 0.0000 \\
\hline
\end{tabular}

\section{Conclusion}

Consumers always consider both the tangible and intangible aspects of products in their purchase decisions. Therefore, a good affective design can attract customers and influence their choices. However, product designers are always faced with the problem of determining the optimal settings of the design attributes for affective design. In this paper, a novel guided search GA approach was proposed to generate the optimal design attribute settings. The proposed approach applies constraints and guided search operators, which are formulated based on mined rules to guide the GA search and to achieve desirable solutions. A case study on the affective design of mobile phones was conducted to illustrate the proposed approach, and a number of validation tests were performed to evaluate their effectiveness. The results of the tests indicate that the guided search GA approach outperforms the GA approach without the guided search strategy in terms of better GA convergence and shorter total computational time. Future studies can focus on the development of a guided search MOGA approach to solve multi-objective optimization problems.

\section{References}

Aktar Demirtas, E., Anagun, A. S., and Koksal, G. (2009). Determination of optimal product styles by ordinal logistic regression versus conjoint analysis for kitchen faucets. International Journal of Industrial Ergonomics, 39(5), 866-875. 
Agrawal, R. B., Deb, K., Deb, K., and Agrawal, R. B. (1995). Simulated binary crossover for continuous search space. Complex Systems, 9, 115-148.

Barnes, C., and Lillford, S. P. (2007). Affective design decision-making-issues and opportunities. CoDesign: International Journal of CoCreation in Design and the Arts, 3(Suppl. 1), 135.

Barnes, C., and Lillford, S. P. (2009). Decision support for the design of affective products. Journal of Engineering Design, 20(5), 477.

Barone, S., Lombardo, A., and Tarantino, P. (2007). A weighted logistic regression for conjoint analysis and Kansei engineering. Quality and Reliability Engineering International, 23(6), 689-706.

Catalano, C. E., Falcidieno, B., Giannini, F., and Monti, M. (2002). A Survey of ComputerAided Modeling Tools for Aesthetic Design. Journal of Computing and Information Science in Engineering, 2(1), 11-20.

Creusen, M. E. H., and Schoormans, J. P. L. (2005). The Different Roles of Product Appearance in Consumer Choice. Journal of Product Innovation Management, 22(1), 6381.

Crilly, N., Moultrie, J., and Clarkson, P. J. (2004). Seeing things: consumer response to the visual domain in product design. Design Studies, 25(6), 547-577.

Chakraborty, I., Kumar, V., Nair, S.B. and Tiwari, R. (2003). Rolling elements bearing design through genetic algorithms. Engineering optimization, 35(6), 649-659.

Chen, C.-H., Khoo, L. P., and Yan, W. (2006). An investigation into affective design using sorting technique and Kohonen self-organising map. Advances in Engineering Software, 37(5), 334-349.

Demirbilek, O., and Sener, B. (2003). Product design, semantics and emotional response. Ergonomics, 46(13), 1346-1360.

D'Souza, Bryan and Simpson, Timothy W.(2003) A genetic algorithm based method for product family design optimization, Engineering Optimization, 35(1), 1-18.

Deb, K., Pratap, A., Agarwal, S., and Meyarivan, T. (2002). A fast and elitist multiobjective genetic algorithm: NSGA-II. IEEE Transactions on Evolutionary Computation, 6(2), 182197.

Fung, K.Y., Kwong, C.K., M. Siu and Yu, K.M., (2012) A Multi-objective Genetic Algorithm Approach to Rule Mining for Affective Product Design, Expert Systems With Applications, 39(8), 7411-7419.

Hsiao, S.-W., and Liu, M. C. (2004). A morphing method for shape generation and image prediction in product design. Design Studies, 23(6), 533-556.

Hsiao, S.-W., and Tsai, H.-C. (2005). Applying a hybrid approach based on fuzzy neural network and genetic algorithm to product form design. International Journal of Industrial Ergonomics, 35(5), 411-428. 
Hsiao, S.-W., and Liu, M. C. (2002). A morphing method for shape generation and image prediction in product design. Design Studies, 23(6), 533-556.

Jiao, J., Zhang, Y., and Helander, M. (2006). A Kansei mining system for affective design. Expert Systems with Applications, 30(4), 658-673.

Jiao, R. J., Xu, Q., Du, J., Zhang, Y., Helander, M., Khalid, H. M., et al. (2008). Analytical affective design with ambient intelligence for mass customization and personalization. International Journal of Flexible Manufacturing Systems, 19(4), 570-595.

Jiao, J. (Roger), Zhang, Y., and Wang, Y. (2007). A heuristic genetic algorithm for product portfolio planning. Computers \& Operations Research, 34(6), 1777-1799.

Khalid, H. M., and Helander, M. G. (2004). A framework for affective customer needs in product design. Theoretical Issues in Ergonomics Science, 5(1), 27.

Kim, H.-S., and Cho, S.-B. (2000). Application of interactive genetic algorithm to fashion design. Engineering Applications of Artificial Intelligence, 13(6), 635-644.

Kwong, C. K., Wong, T. C., and Chan, K. Y. (2009). A methodology of generating customer satisfaction models for new product development using a neuro-fuzzy approach. Expert Systems with Applications, 36(8), 11262-11270.

Kaplan, S., and Rabadi, G. (2013). Simulated annealing and metaheuristic for randomized priority search algorithms for the aerial refueling parallel machine scheduling problem with due date-to-deadline windows and release times. Engineering Optimization, 45(1), 67-87.

Kuang, J. and Jiang, P. (2008) Product platform design for a product family based on Kansei engineering, Journal of Engineering Design, 20(6), 589-607.

Lai, H. H., Chang, Y. M., and Chang, H. C. (2005a). A robust design approach for enhancing the feeling quality of a product: a car profile case study. International Journal of Industrial Ergonomics, 35(5), 445-460.

Liu, Y. (2003). Engineering aesthetics and aesthetic ergonomics: Theoretical foundations and a dual-process research methodology. Ergonomics, 46(13), 1273-1292.

Lin, Y.-C., Yeh, C.-H., and Hung, C.-H. (2008). A Neural Network Approach to the Optimal Combination of Product Color Design. Proceedings, Fourth International Conference on Networked Computing and Advanced Information Management, 2008, 1, 53-57.

Mishra, E. A. K., Mohapatra, E. Y., and Mishra, E. A. K. (2013). Multi-Objective Genetic Algorithm: A Comprehensive Survey. International Journal of Emerging Technology and Advanced Engineering, 3(2), 81-90.

Nagamachi, M. (2002). Kansei engineering as a powerful consumer-oriented technology for product development. Applied Ergonomics, 33(3), 289-294.

Nagamachi, M. (1995). Kansei Engineering: A new ergonomic consumer-oriented technology for product development. International Journal of Industrial Ergonomics, 15(1), 3-11. 
Nagamachi, M. (2008). Perspectives and the new trend of Kansei/affective engineering. The TQM Journal, 20(4), 290-298.

Noble, C. H., and Kumar, M. (2008). Using product design strategically to create deeper consumer connections. Business Horizons, 51(5), 441-450.

Park, J., and Han, S. H. (2004). A fuzzy rule-based approach to modeling affective user satisfaction towards office chair design. International Journal of Industrial Ergonomics, 34(1), 31-47.

Saridakis, K. M., and Dentsoras, A. J. (2008). Soft computing in engineering design - A review. Advanced Engineering Informatics, 22(2), 202-221.

Schütte, S., and Eklund, J. (2005). Design of rocker switches for work-vehicles--an application of Kansei Engineering. Applied Ergonomics, 36(5), 557-567.

Sivakumar, R. (2012). A performance study of real coded genetic algorithm on gear design optimization. Advanced Material Research, 622-623, 64-68.

Velasco, N., Dejax, P., Gueret, C., and Prins, C. (2012). A non-dominated sorting genetic algorithm for a bi-objective pick-up and delivery problem. Engineering Optimization, 44(3), 305-325.

Yanagisawa, H., and Fukuda, S. (2005). Interactive reduct evolutional computation for aesthetic design. Journal of Computing and Information Science in Engineering, 5(1), 1-7.

Yang, C.-C., and Shieh, M.-D. (2010). A support vector regression based prediction model of affective responses for product form design. Computers \& Industrial Engineering, 59(4), 682-689. 